\title{
Peripheral cemento- ossifying fibroma
}

N Ravi ${ }^{1}$ and Shyam Prasad Reddy D²

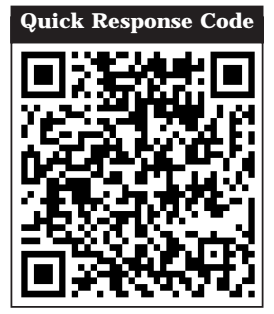

doi: $10.5866 / 2015.7 .10200$

1Post-Graduate Student

${ }^{2}$ Reader

Department of Oral \& Maxillofacial Pathology

Kamineni Institute of Dental Sciences,

Narketpally, Nalgonda (Dist),

Telangana, India

\section{Article Info:}

Received: J uly 8, 2015

Review Completed: August 9, 2015

Accepted: September 10, 2015

Available Online: J uly, 2015 (www.nacd.in)

(C) NAD, 2015 - All rights reserved

Email for correspondence:

shyamprasadreddy_d@yahoo.co.in

\begin{abstract}
:
Peripheral cemento-ossifying fibroma (PCOF) is a reactive gingival overgrowth occurring frequently in the maxillary anterior region in teenagers and young adults. It is a slowgrowing benign tumor with non odontogenic origin which may lead to pathologic migration and other periodontal problems. The recurrence rate of peripheral cemento-ossifying fibroma is reported to be $8 \%$ to $20 \%$, The lesion is asymptomatic, firm, pinkish red \& pedund uated, histol ogical ly shows cellular fibrous connective tissue stroma with calcified osseous \& cementum like calcifications. The present PCOF case report comprises the growth that occurred in a 12 year old male child in the mandibular anterior region.
\end{abstract}

Key words: Fibroma, gingival overgrowth, peripheral cemento-ossifying fibroma.

\section{Introduction:}

Ossifying fibroma occurs mostly in craniofacial bones and categorized into two types central and peripheral. The central type of ossifying fibroma arises from the endosteum or the periodontal ligament adjacent to the root apex and expands from the medullary cavity of the bone, and the peripheral type occurs on the soft tissues overlying the alveolar process. ${ }^{1}$ Ossifying fibroma is a benign neoplasm composed of proliferating fibroblasts with osseous products that include bone and ovoid calcifications; and the lesions are well demarcated from the adjacent bone.
Many types of localized reactive lesions may occur on the gingiva, including focal fibrous hyperplasia, pyogenic granuloma, peripheral giant cell granuloma and peripheral cemento-ossifying fibroma (PCOF). ${ }^{2}$ It is widely considered that PCOF originates from the cells of the periodontal ligament, and is often associated with trauma or local irritants, such as subgingival calculas, plaque, poor-quality dental restorations and dental appliances. ${ }^{3}$ Clinically, PCOF's are sessile or pedunculated, with ulcerated, and erythematous. Most lesions are less than $2 \mathrm{~cm}$ in size, although larger ones occasionally occur. ${ }^{3}$ It is more commonly seen in the first and 
second decades of life and has a female preponderance. There is a slight predilection for the maxillary arch (60\%) and the incisor cuspid region (50\%). ${ }^{4}$ The present case report comprised a growth that occurred in a 12 year old male child in the mandibular anterior region which was diagnosed as PCOF.

\section{CASE REPORT}

A 12 year old male child reported with a chief complaint of growth on lower teeth gums of anterior region since two months. On intra oral examination a well defined solitary sessile growth of size $1 \times 1 \mathrm{~cm}$ was seen at interdental papilla in relation to 31 and 41. The lesion was extending anterio posteriorly from distal third of 31 to middle third of 41 ; and superio inferiorly from occulsal plane to attached gingiva of 31and 41 (Figure 1). The lesion was also extending to lingual side through inter-dental gap of 31 and 41 (Figure 2). The lesion was soft in consistency tender on palpation with bleeding on probing.

Patient was advised routine hematological and radiographic investigations, where blood investigations were with in the normal range and no significant changes were observed in the radiograph. Based on these clinical and radiographic findings provisional diagnosis was made as pyogenic granuloma.

Under local anesthesia, the whole growth was excised and sent for histopathological examination. Sections were stained with routine $\mathrm{H}$ and $\mathrm{E}$. Microscopic examination revealed hyperplastic parakeratotic stratified squamous epithelium of variable thickness (Figure 3).The underlying connective tissue stroma comprised of chronic inflammatory cell infiltrate and irregular mineralization foci (Figure 4) along with numerous spindle shaped fibroblasts. The cells were arranged in a circular shape around irregular mineralization foci .The calcified areas resembled cementum-like and bone-like ossifying areas (Figure 5). Clinical assessment and histopathol ogic report confirmed the diagnosis as peripheral cement-ossifying fibroma (PCOF).

\section{Discussion :}

Peripheral Ossifying Firoma (POF) was first reported by the Shepherd in 1844 as alveolar exostosis. Menzel first described the lesion ossifying fibroma in 1872, but its terminology was given by Montgomery in $1927 .{ }^{5}$ Eversol and Robin in 1972, later coined the term peripheral ossifying fibroma. ${ }^{2}$ Peripheral-Ossifying Fibroma is a non-neoplastic reactive growth usually arise from the interdental papilla of gingiva. Various nomenclature are given for the POF by different authors, such as peripheral cementifying fibroma, ossifying fibro-epithelial polyp, peripheral fibroma with cementogenesis, peripheral fibroma with osteogenesis, peripheral fibroma with calcification, calcifying fibroblastic granuloma and calcifying or ossifying fibrous epulis. ${ }^{2,}{ }^{6}$ When bone predominates the term 'Ossifying' is used and when curvilinear trabeculae or spheroidal calcifications are seen the term 'cementifying' is used and when bone and cementumlike tissues are observed, the lesions have been referred to as cemento-ossifying fibroma in nomenclature. $^{7}$

Microscopic examination of $\mathrm{H}$ and $\mathrm{E}$ stained section reveals stratified squamous epithelium of variable thickness with underlying connectivetissue stroma comprising of irregular mineralization foci al ong with numerous spindle shaped fibroblasts. The cells will be arranged in a circular shape around irregular mineralization foci and the calcified areas resemble cementum-like and bone-like ossifying areas. The present case report also confirms the presence of irregular mineralization foci along with numerous spindle shaped fibroblasts. The cells were arranged around irregular mineralization foci and the calcified areas resemble cementum-like and bone-like ossifying areasdiagnosis as peripheral cement-ossifying fibroma (PCOF).

It is a fairly common lesion, comprising nearly $3 \%$ of oral lesions. The site of occurrence of POF is usually anterior to molars in both maxilla and mandible equally, and in more than $50 \%$ of cases in theincisor, and canine regions. ${ }^{2}$ ThePOF has a peak incidence in young and teenaged females. Cundiff reported that the lesion is prevalent between ages 


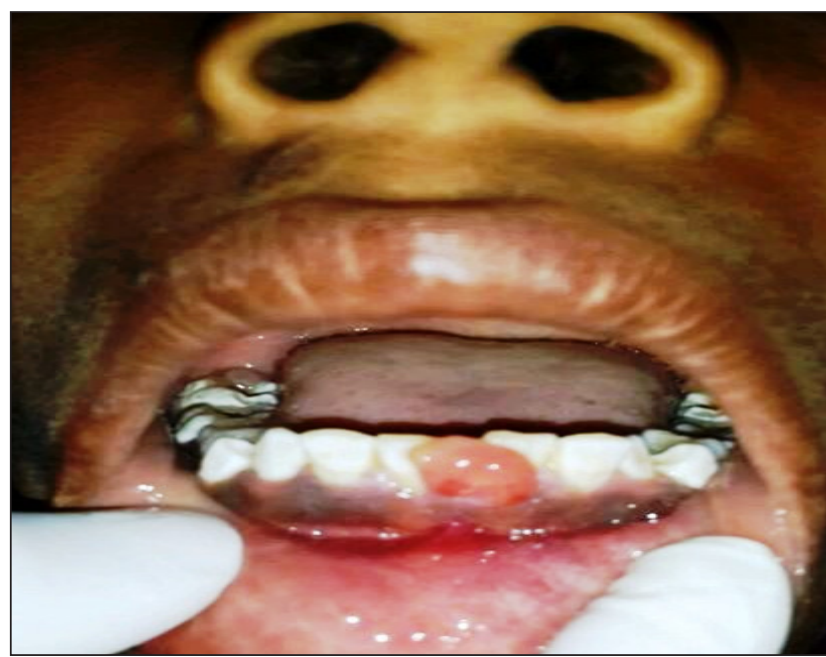

Figure 1: Clinical photograph showing solitary sessile growth of size $1 \times 1 \mathrm{~cm}$ at inter-dental papilla of 31 and 41 .

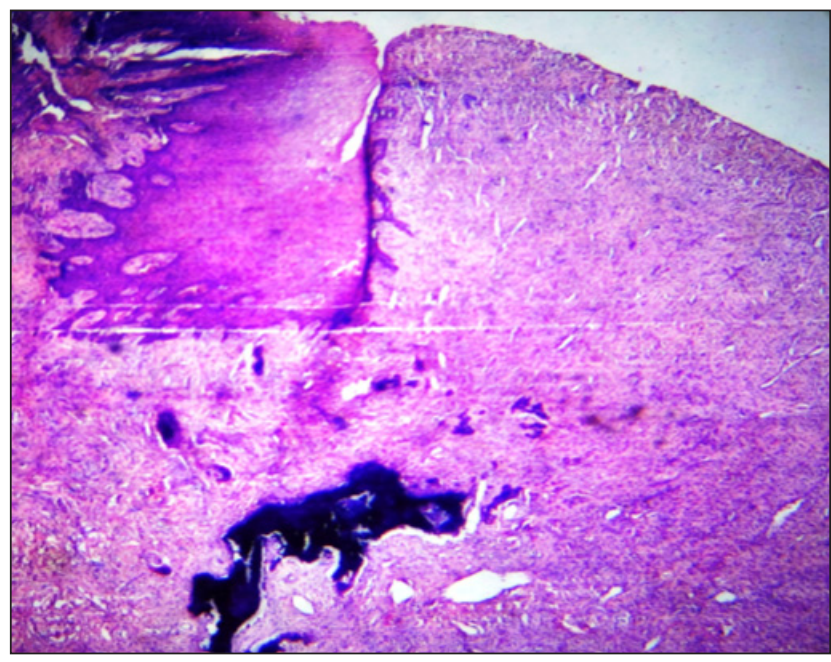

Figure 3: $\mathrm{H}$ and $\mathrm{E}$ stained section showing hyperplastic parakeratotic stratified squamous epithelium with variable thickness (x4).

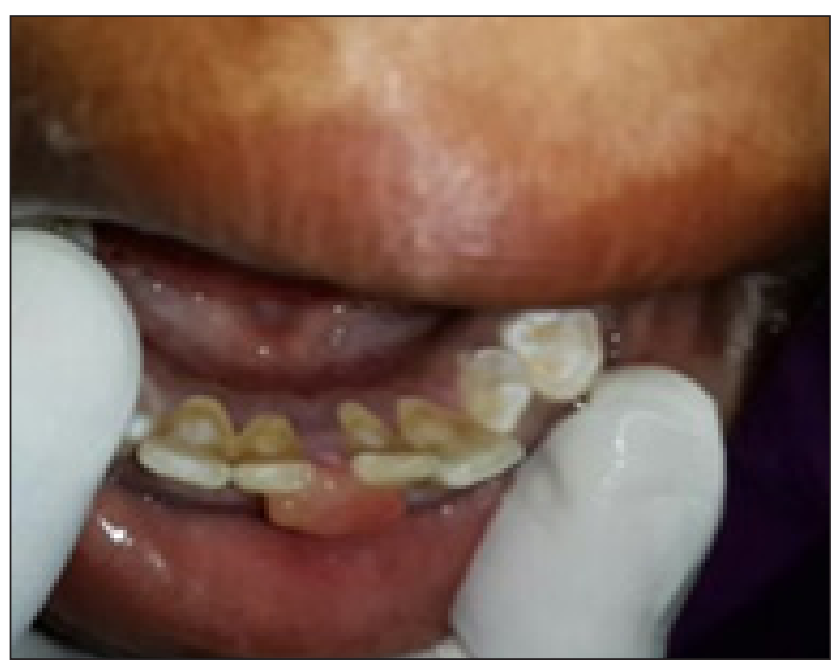

Figure 2: Clinical photograph showing lesion extending lingually through interdental gap of 31 and 41.

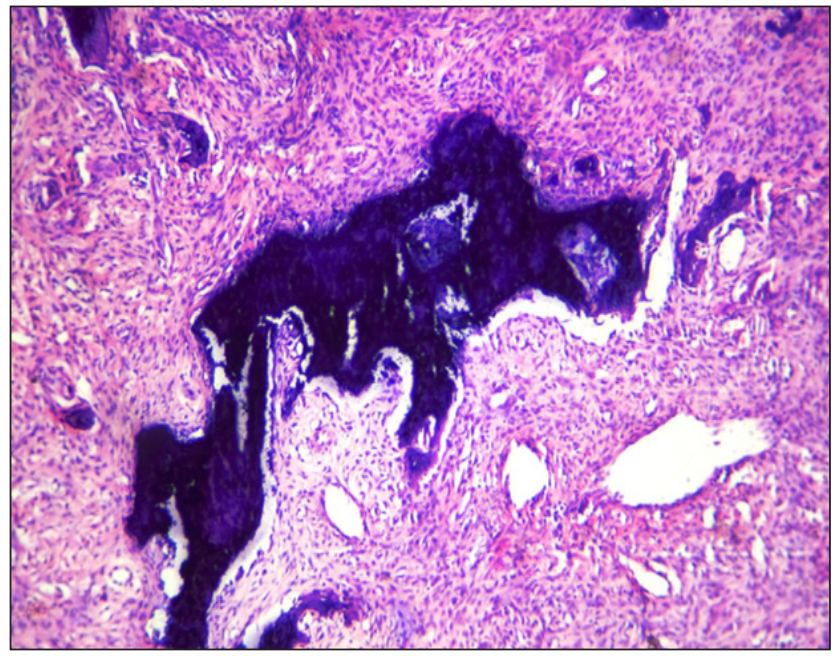

Figure 4: $\mathrm{H}$ and $\mathrm{E}$ stained section showing chronic inflammatory cell infiltrate and irregular mineralization foci (x10).

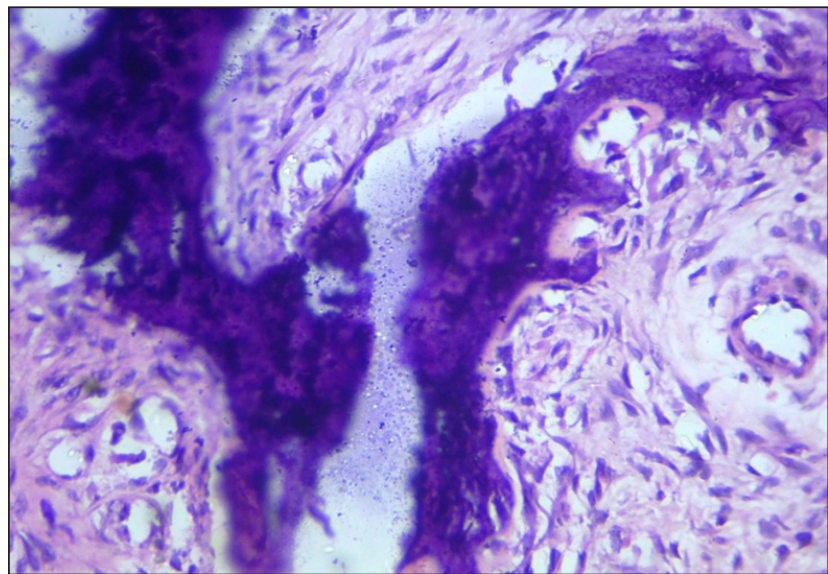

Figure 5: $\mathrm{H}$ and $\mathrm{E}$ stained section showing fibroblasts arranged in a circular shape around irregular mineralization foci. The calcified areas resemble cementum-like and bone-like ossifying areas (x40). 
of 5 and 25 years, with a peak incidence at 13 years of age and he also reported a definite female predilection. Female to male ratio may vary from 2:1 to 3:2. ${ }^{2}$ Excessive proliferation of mature fibrous connective tissue is a response to gingival injury, gingival irritation, subgingival calculus or a foreign body in the gingival sulcus.

Two schools of thought exist regarding histogenesis of POF which are controversial:

1) POF may initially develop as pyogenic granuloma that undergoes subsequent fibrous maturation and calcification. It represents the progressive stage of the same spectrum of pathogenesis.

2) POF is due to inflammatory hyperplasia of cells of periodontal ligament/periosteum. Metaplasia of the connective tissue leads to dystrophic calcification and bone formation. ${ }^{8}$

Lesions involving the gingival soft tissues are rare when compared to the lesions appearing within bone. Mesquita RA found higher numbers of Argyrophilic Nucleolar Organizer Regions (AgNORs) and proliferating cell nuclear antigen (PCNA)-positive cells in ossifying fibroma than in peripheral ossifying fibroma indicating higher proliferative activity in ossifying fibroma, X-ray diffraction analysis indicated that the mineral phase of both central and peripheral tissues consists of apatite crystals and that the crystallinity of these apatites is lower than that of bone apatite. It was also suggested that the crystallinity of the apatites might improve progressively with the development of the lesion, possibly to the same degree as that of bone apatite. $^{9}$

An attempt has been made by Endo et al. to distinguish cementifying fibromas from ossifying fibromas and fibrous dysplasias by using immunohistochemical analysis for keratan sulfate and chondroitin-4-sulfate, in which the cementifying fibromas showed significant immuno-reactivity for keratan sulfate, and ossifying fibromas and fibrous dysplasias showed intensive immunostaining for chondroitin-4-sulfate. ${ }^{7}$

\section{Conclusion:}

PCOF is a slowly progressivelesion with limited growth exclusively occurring on gingiva. ${ }^{7}$ Clinically, it is difficult to differentiate between most of the reactive gingival lesions particularly in the initial stages. It is important to eliminate the etiological factors and the tissue has to be histopathologically examined for accurate diagnosis and management. Treatment suggests surgical excision, including the periosteum and scaling of adjacent teeth. 7,8

\section{References:}

1. Miller CS, Henry RG, Damm DD. Proliferative mass found in gingiva. J Am Dent Assoc 1990; 121(4):559-560.

2. Mahavir B Mishra, Kundendu Arya Bhishen, Shanu Mishra. Case Report- Peripheral ossifying fibroma. J Oral Maxillofacial Path 2011; 15(1): 65-68.

3. Kumar SK, Ram S, J orgensen MG, Shuler CF, Sedghizadeh PP. Multicentric peripheral ossifying fibroma. J Oral Sci 2006; 48(4): 239-243.

4. Poonacha KS, Anand L Shigli, Dayanand shirol. Peripheral ossifying fibroma: A clinical report. Contemp Clin Dent 2010; 1(1): 54-56.

5. Fayiza Yaqoob Khan, Suhail Majid J an, Mubashir Mushtaq. Multicentric peripheral ossifying fibroma: A case report and review of the literature. J Ind Soc Periodontol 2013; 17(5):648-652.

6. Parveen Dahiya, Reet Kamal,Gaurav Saini, and Shweta Agarwal. Case report- Peripheral ossifying fibroma. J Nat Sci Biol Med 2012; 3(1):94-96.

7. Anirban Chatterjee, Neha Ajmera, Amit Singh. Case report Peripheral cemento-ossifying fibroma of maxilla. J Indian Soc Periodontol 2010; 14(3):186-189.

8. Prasad S, Reddy SB, Patil SR, Kalburgi NB, Puranik RS. Peripheral ossifying fibroma and pyogenic granuloma. Are they interrelated? N Y State Dent J 2008; 74:50-52.

9. Renu Yadav, Anubhagulati. Peripheral ossifying fibroma; a case report. J Oral Sci 2011; 51(1):151-154.

\section{Gain quick access to our journal online View our journal at www.nacd.in}

